\title{
BMJ Open HORYZONS trial: protocol for a randomised controlled trial of a moderated online social therapy to maintain treatment effects from first- episode psychosis services
}

\author{
Mario Alvarez-Jimenez, ${ }^{1,2}$ Sarah Bendall, ${ }^{1,2}$ Peter Koval, ${ }^{3}$ Simon Rice, ${ }^{1,2}$ \\ Daniela Cagliarini, ${ }^{1,2}$ Lee Valentine, ${ }^{1,2}$ Simon D'Alfonso, ${ }^{1,4}$ Christopher Miles, ${ }^{1,2}$ \\ Penni Russon, ${ }^{1,2}$ David L Penn, ${ }^{5,6}$ Jess Phillips, ${ }^{1,2}$ Reeva Lederman, ${ }^{4}$ Greg Wadley, ${ }^{4}$ \\ Eoin Killackey, ${ }^{1,2}$ Olga Santesteban-Echarri, ${ }^{7}$ Cathrine Mihalopoulos, ${ }^{8}$ \\ Helen Herrman, ${ }^{1,2}$ Cesar Gonzalez-Blanch, ${ }^{9}$ Tamsyn Gilbertson, ${ }^{1,2}$ Shalini Lal, ${ }^{10,11,12}$ \\ Richard Chambers, ${ }^{13}$ Rothanthi Daglas-Georgiou, ${ }^{1,2}$ Cristina Latorre, ${ }^{14}$ \\ Sue M Cotton, ${ }^{1,2}$ Patrick D McGorry, ${ }^{1,2}$ John F Gleeson ${ }^{5}$
}

To cite: Alvarez-Jimenez M, Bendall S, Koval P, et al. HORYZONS trial: protocol for a randomised controlled trial of a moderated online social therapy to maintain treatment effects from first-episode psychosis services. BMJ Open 2019:9:e024104. doi:10.1136/ bmjopen-2018-024104

- Prepublication history for this paper is available online. To view these files, please visit the journal online (http://dx.doi. org/10.1136/bmjopen-2018024104).

Received 20 June 2018 Revised 9 0ctober 2018 Accepted 10 October 2018

D Check for updates

(c) Author(s) (or their employer(s)) 2019. Re-use permitted under CC BY-NC. No commercial re-use. See rights and permissions. Published by BMJ.

For numbered affiliations see end of article.

\section{Correspondence to}

Professor Mario Alvarez-

Jimenez;

mario.alvarez@orygen.org.au

\section{ABSTRACT}

Introduction Specialised early intervention services have demonstrated improved outcomes in first-episode psychosis (FEP); however, clinical gains may not be sustained after patients are transferred to regular care. Moreover, many patients with FEP remain socially isolated with poor functional outcomes. To address this, our multidisciplinary team has developed a moderated online social media therapy (HORYZONS) designed to enhance social functioning and maintain clinical gains from specialist FEP services. HORYZONS merges: (1) peer-to-peer social networking; (2) tailored therapeutic interventions; (3) expert and peer-moderation; and (4) new models of psychological therapy (strengths and mindfulness-based interventions) targeting social functioning. The aim of this trial is to determine whether following 2 years of specialised support and 18-month online social media-based intervention (HORYZONS) is superior to 18 months of regular care.

Methods and analysis This study is a single-blind randomised controlled trial. The treatment conditions include HORYZONS plus treatment as usual (TAU) or TAU alone. We recruited 170 young people with FEP, aged 16-27 years, in clinical remission and nearing discharge from Early Psychosis Prevention and Intervention Centre, Melbourne. The study includes four assessment time points, namely, baseline, 6-month, 12-month and 18-month follow-up. The study is due for completion in July 2018 and included a 40-month recruitment period and an 18-month treatment phase. The primary outcome is social functioning at 18 months. Secondary outcome measures include rate of hospital admissions, cost-effectiveness, vocational status, depression, social support, loneliness, self-esteem, self-efficacy, anxiety, psychological well-being, satisfaction with life, quality of life, positive and negative psychotic symptoms and substance use. Social functioning will be also assessed in real time through our Smartphone Ecological Momentary Assessment tool.
Strengths and limitations of this study

This is the first randomised controlled trial to evaluate the effectiveness of an online intervention designed to extend the benefits of specialised early psychosis services.

- HORYZONS is the first intervention to harness online social media technology and use strengths and mindfulness-based interventions to improve longterm recovery in early psychosis.

- HORYZONS was developed by a multidisciplinary team in partnership with young people, with the purpose of being scalable across, and embedded within, early intervention services.

- In line with recent clinical trials evaluating extended models of care for early psychosis services, the control intervention consists of routine care as opposed to a placebo intervention accounting for increased attention and unspecific therapeutic factors.

- Due to the nature of psychosocial interventions, participants and clinicians were not blind to treatment allocation.

Ethics and dissemination Melbourne Health Human Research Ethics Committee (2013.146) provided ethics approval for this study. Findings will be made available through scientific journals and forums and to the public via social media and the Orygen website.

Trial registration number ACTRN12614000009617; Preresults.

\section{INTRODUCTION}

Psychosis can be a devastating mental health disorder. Onset is often in adolescence and early adulthood and in many cases 
follows a chronic and relapsing course that results in great personal suffering and societal costs. ${ }^{1}{ }^{2}$ Against this daunting picture, early intervention is now seen as the most promising and evidence-based approach to improve the long-term outcomes of psychosis. ${ }^{3}$ Specialist first-episode psychosis (FEP) services originated in the early 1990s with a focus on reducing treatment delays, providing youth friendly, phase-specific support and preventing the development of long-term functional and social disability. ${ }^{3}$ Over the past two decades, several randomised controlled trials (RCTs) conducted across countries and mental health systems have demonstrated that these services improve psychotic symptoms, reduce relapse rates, foster patient satisfaction and result in tangible economic benefits. ${ }^{4-8}$

There are limits, however, to the impact of early intervention services. First, specialist FEP services typically have treatment resources for 2 years, and recent reports indicate that the benefits of early intervention seen at the end of 2 years may not persist at 3 years postdischarge. ${ }^{9} 10$ Second, even after receiving specialised services, functional recovery lags behind symptomatic remission, and many young people with FEP experience significant social functioning deficits and poor quality of life. ${ }^{11}$ Indeed, the onset of psychosis has been characterised as a 'social network crisis', ${ }^{12}$ which is not improved by early intervention services. Young people with psychosis have smaller social networks, fewer people to turn to in a crisis, ${ }^{13}$ are between nine and nine times less likely to have confidants compared with their peers ${ }^{14}$ and report on average 2-3 lonely days per week. ${ }^{15}$ Smaller social networks and lower perceived social support are, in turn, predictive of poorer long-term functional outcomes, shorter time in remission and increased hospital admissions. ${ }^{16-18}$ Taken together, these research findings underscore the need for new treatment approaches that extend the benefits of early intervention services and, ultimately, promote long-term social recovery.

While difficulties with social functioning are commonplace following FEP and can lead to poor long-term outcomes, very few studies have assessed interventions targeting social functioning as a primary outcome. The most researched psychological intervention for FEP has been cognitive behavioural therapy, which is primarily focused on reducing the positive symptoms of psychosis. ${ }^{19}$ Recognising this gap, a recent trial evaluated a social recovery therapy in combination with early intervention services to enhance social recovery in FEP. ${ }^{19}$ Study results showed an improvement in structured activity in those receiving the intervention relative to those receiving early intervention services alone. The renewed focus on social recovery is also consistent with recent psychological models, which have proposed self-efficacy ${ }^{20} 21$ and positive emotions ${ }^{22}$ as important targets to promote social functioning in psychosis. Strengths-based and mindfulness-based interventions have been put forward as key interventions to increase self-efficacy and positive emotions, ${ }^{23}$ respectively, with preliminary studies supporting their potential to improve social functioning in psychosis. ${ }^{24}$

A complementary approach to improving long-term recovery in FEP is to extend the duration of specialised treatment. ${ }^{26} 27$ This view is underpinned by findings that the first 5 years after psychosis onset constitute a critical period, determining longer term outcomes. ${ }^{27}{ }^{28}$ Similarly, promoting sustained social and functional recovery in the early course of psychosis appears to be a key path towards long-term functional recovery. ${ }^{29}$ Two recent RCTs have evaluated the effects of the current model of early intervention (ie, 2 years of specialised treatment) versus an extended model of care (ie, 5 years of specialised treatment ${ }^{3031}$ with mixed results. In one of these trials, the extended model of care improved length of remission of positive and negative symptoms relative to regular care. ${ }^{31}$ Conversely, a second study showed no significant improvements in clinical or social outcomes associated with the extended model of care. ${ }^{30}$ An additional clinical trial examined the effects of prolonging the period of specialised care for 12 months (ie, 3 years vs 2 years of specialised treatment). ${ }^{32}$ This study showed significant improvements in functional outcomes at the end of the 3-year compared with 2-year specialised support. However, treatment benefits were not sustained, with no significant differences across treatment groups at 1 and 2 years postspecialised intervention. ${ }^{32}$

An alternative to prolonging the duration of specialised intervention is to offer extended, lower intensity maintenance treatment following the first 2 years of specialised treatment. ${ }^{27}$ This is supported by findings that the termination of the specialised intervention and transfer of care brings about feelings of loss for the patients ${ }^{9}$ and significantly derails engagement with treatment services, ${ }^{33}$ a pivotal element of early intervention programmes. Thus, a lower intensity level of care may bridge the gap between specialised intervention and standard treatment and provide a cost-effective alternative to bring about sustained benefits in FEP. This approach has shown promising results in a single group study, with improvements seen at 2 years (ie, end of specialised care) being maintained at 5 years (ie, after 3 years of lower intensity specialised treatment). ${ }^{27}$

Online-based and mobile-based interventions can also provide a lower intensity, cost-effective and engaging approach to prolonging the benefits of specialised FEP services. Indeed, the extant research shows that online interventions are feasible, acceptable and may improve a range of important domains in psychosis treatment including psychotic symptoms, hospital admissions, social connectedness and depression. ${ }^{34}{ }^{35}$ However, most studies conducted to date have employed uncontrolled designs, were underpowered, included short follow-up periods, targeted people with chronic schizophrenia, did not use online social media and did not specifically target social functioning. ${ }^{34}$ To the best of our knowledge, only one pilot study has evaluated the acceptability and preliminary benefits of an online intervention in young people with FEP. ${ }^{36}$ 
Finally, online social networks provide a particularly promising avenue to foster social functioning in young people with FEP. A recent study revealed that $89 \%$ of young people aged 18-29 years use social media daily, ${ }^{37}$ a frequency that is on the rise. ${ }^{38}$ Use of online social media has been associated with increased life satisfaction, ${ }^{39}$ self-esteem ${ }^{39}$ and social capital, ${ }^{40}$ as well as lower loneliness and depression, ${ }^{41}$ particularly for those who post content to the social network and are active users. ${ }^{42}$ Recent surveys indicate that social media habits of young people with psychosis resemble that of their peers: virtually all regularly use social media, on average 10 times and 2 hours per day. ${ }^{43}{ }^{44}$ Particularly relevant to the therapeutic potential of social media in FEP, $78 \%$ would like to obtain help from clinicians via social media, $40 \%$ increase their use of social media when experiencing symptoms ${ }^{43}$ and the majority strongly agree with using social media as a platform from mental health support. ${ }^{45}$ Thus, coupled with psychological interventions specifically addressing social recovery such as strengths-based and mindfulness-based approaches, social media provides an opportunity deliver acceptable, extended lower intensity support with potential to foster long-term social functioning in FEP.

\section{Aims and hypotheses}

The objective of this trial was to determine whether extending the treatment period of a specialised FEP service through an 18-month, step-down, novel online social media-based intervention (HORYZONS) produces better outcomes compared with 2 years of specialist FEP treatment followed by treatment as usual (TAU), using a randomised controlled single-blind design. An additional aim of this trial is to determine the cost-effectiveness of HORYZONS.

The primary hypothesis is that, relative to TAU, HORYZONS will lead to improved social functioning at 18 months among young people with FEP. The secondary hypotheses are that, relative to TAU, HORYZONS will reduce the rate of hospital admissions due to psychotic symptoms and lead to improvements in depression, vocational outcomes, satisfaction with life, social support, loneliness, self-esteem, self-efficacy, anxiety, stress, positive and negative psychotic symptoms, psychological well-being, quality of life and substance use. Finally, we hypothesise that HORYZONS will be more cost-effective than TAU.

\section{METHODS AND ANALYSIS \\ Study design}

The study design is an 18-month, parallel groups, singleblind, RCT in which 170 participants with remitted FEP have been allocated to either the current mainstream model of early intervention for psychosis (ie, 2 years of specialised treatment followed by discharge to TAU), or TAU in tandem with a moderated online social media intervention (HORYZONS), for 18 months.
The design includes four assessment time points: baseline, 6 months, 12 months and 18 months. The RCT includes a 40-month recruitment period and an 18-month treatment phase, with the study being completed within 5 years. The protocol development addressed all aspects of Good Clinical Practice, ${ }^{46}$ Consolidated Standards of Reporting Trials EHEALTH criteria ${ }^{47}$ and Standard Protocol Items: Recommendations for Interventional Trials guidelines. ${ }^{48}$

\section{Setting}

Recruitment of the trial participants commenced in October 2013 and finalised in January 2017 at Early Psychosis Prevention and Intervention Centre (EPPIC), a subprogramme of Orygen Youth Health, Melbourne. EPPIC is a publicly funded specialist FEP programme servicing 250 new referrals for FEP per year. EPPIC provides 18 months-2 years of specialised care after which patients are discharged and transferred to TAU. ${ }^{49}$ Follow-up assessments will be concluded in July 2018.

\section{Participants}

Inclusion criteria for participants were: (A) a first episode of a Diagnostic and Statistical Manual of Mental Disorders (DSM-IV) psychotic disorder or mood disorder with psychotic features; (B) aged 16-27 years inclusive; (c) $\leq 6$ months treatment with an antipsychotic medication prior to registration with EPPIC; (D) remission of positive symptoms of psychosis, defined, using the Positive and Negative Syndrome Scale (PANSS), ${ }^{50}$ as 4 weeks or more of scores of 3 (mild) or below on items P2 (conceptual disorganisation) and G9 (unusual thought content) and scores of 4 (moderate) or below with no functional impairment on items P3 (hallucinatory behaviour) and P1 (delusions). Additional inclusion criteria to ensure low level of risk within HORYZONS included: $(F)$ low aggressiveness, defined by a score of 3 or below on the poor impulse control item of the PANNS for the month prior to study entry; and $(\mathrm{G})$ moderate or lower suicidal risk defined as a score of 4 or below on the suicidality subscale of the Brief Psychiatric Rating Scale - expanded version ${ }^{51}$ for the month preceding study entry. Finally, participants were required to nominate an emergency contact to be eligible for the study.

Exclusion criteria included: (A) intellectual disability; and (B) inability to converse in or read English. Additional exclusion criteria to ensure safety within the online system included (C) a DSM-IV diagnosis of either antisocial personality disorder (ASPD) or (D) borderline personality disorder (BPD) as well as clinical evidence that the BPD features cause interpersonal difficulties in the treatment environment.

The Structured Clinical Interview for DSM-IV Axis I Disorders patient edition (SCID-I/P) ${ }^{52}$ was used as the standardised measure of DSM-IV diagnosis of mental illness. The BPD (13 items) and Conduct Disorder/ASPD (22 items) screening questions of the SCID-II Personality Questionnaire were used to assess for BPD and ASPD. ${ }^{53}$ 
Withdrawal from the trial occurred if: (A) participation in the study interfered with appropriate clinical management of risk of harm to self or others (as judged by the treating clinicians and/or senior researchers); (B) serious adverse events developed that could be associated with the online intervention; and $(\mathrm{C})$ participants failed to comply with the terms of use of the online intervention. Withdrawal from the study could be at the request of the participant or at the discretion of the investigator.

\section{Enrolment and randomisation}

The recruitment and allocation procedures are depicted in figure 1 . The study coordinator liaised with the Orygen Youth Health Quality and Evaluation Unit to obtain a list of young people with FEP nearing discharge from EPPIC. This list was updated every 3 months during the recruitment phase. The study coordinator assessed the initial eligibility of young people within 3 months of discharge in consultation with EPPIC case managers and treating doctors. Clients deemed potentially eligible were approached by the study coordinator to obtain written informed consent. Next, eligibility was confirmed through a screening assessment. Eligible participants completed the baseline assessment and were subsequently randomised to either HORYZONS plus TAU or TAU alone at a ratio of 1:1. Randomisation was carried out remotely according to the International Conference on Harmonization E9 Statistical Principles Guidelines. ${ }^{54}$ An independent statistician created the randomisation sequence using permutated blocks. The study coordinator randomised the participants via a secure online Research Project Management System (RPMS). The RPMS sent an automated email to the study coordinator and investigators notifying them of the outcome of randomisation. Finally, the study coordinator informed the participant of the allocation.

The study assessors undertaking the follow-up assessments are kept blind to treatment allocation via the following mechanisms: (1) at the commencement of each research interview the assessor reminds participants of the importance of the blind, (2) study assessors are excluded from all clinically related discussions regarding participants and (3) the assessors were forbidden from accessing participants' medical records. The assessors record their best guess of participants' treatment allocation at 6-month, 12-month and 18-months follow-up in order to enable an assessment of the success of treatment concealment. Any instances of unblinding were recorded.

\section{INTERVENTIONS \\ HORYZONS}

HORYZONS has been developed by a large multidisciplinary team of researchers, clinical psychologists, programmers, creative writers, graphic artists and experts in human-computer interaction. ${ }^{36}{ }^{55}$ HORYZONS was designed following participatory design principles with the purpose of addressing social functioning in early psychosis. For example, focus groups with young people with psychosis revealed that they favoured a social media-based platform enabling meaningful peer-to-peer contact as well as clinicians' support. ${ }^{35} 56$ In addition, young people called for online interventions focused on promoting personal strengths and self-efficacy as opposed to merely ameliorating symptoms and deficits. Finally, young people indicated that the system should provide self-guided, interactive, tailored interventions, relevant to their changing needs. ${ }^{35} 56$

Informed by young people's continual feedback as well as relevant research in the mental health and human computer interaction fields, ${ }^{55}$ the design of HORYZONS merged: (1) interactive online therapy ('Pathways and Steps'), (2) peer-to-peer online social networking ('the café') and (3) peer and (4) expert moderation. All components of HORYZONS were designed to reinforce each other, creating a flow for the young person between the social and therapy elements. For example, young people are encouraged to post comments and interact with others while engaging with therapy content and are, at the same time, prompted by moderators to practice their strengths or use skills they have learnt while engaging with the social network. Young people can log on to HORYZONS at any time via an Internet-enabled desktop or mobile device.

\section{Interactive online therapy modules ('pathways and steps')}

HORYZONS integrates a number of online 'pathways' organised into distinct themes including: understanding psychosis, identifying and exercising personal strengths, promoting positive connections with others, fostering positive emotions, early warning signs and prevention of relapse, managing stress and anxiety, dealing with depression and vocational skills. With the aim of increasing the usability and take-up of therapeutic content, pathways consist of thematically related interactive therapy 'Steps'. The online 'Steps' are discrete, interactive, evidencebased therapy modules primarily targeting social functioning in young people with psychosis, for example, through fostering self-efficacy (eg, identifying personal strengths via an interactive card sort game based on the strengths-based framework ${ }^{36}$ ), positive emotions and subjective well-being (eg, practising mindfulness and self-compassion), or positive connections with others (eg, illustrating how to respond empathically to others). The content of the Steps was informed by previous studies linking use of personal strengths, increased self-efficacy and positive emotions with improved social functioning in psychosis. ${ }^{21} 222557$ Online Steps further address comorbid symptoms such as anxiety and depression as well as vocational support (informed by our previous work ${ }^{58}$ ). Finally, the design of HORYZONS and therapeutic content was strongly influenced by self-determination theory, an empirically supported theory of motivation which focuses on the processes and social environments that facilitate or hamper social functioning. ${ }^{59}$

The Steps incorporate prompts for participants to share their thoughts and reactions to the therapeutic material with other users through embedded 'Talking 


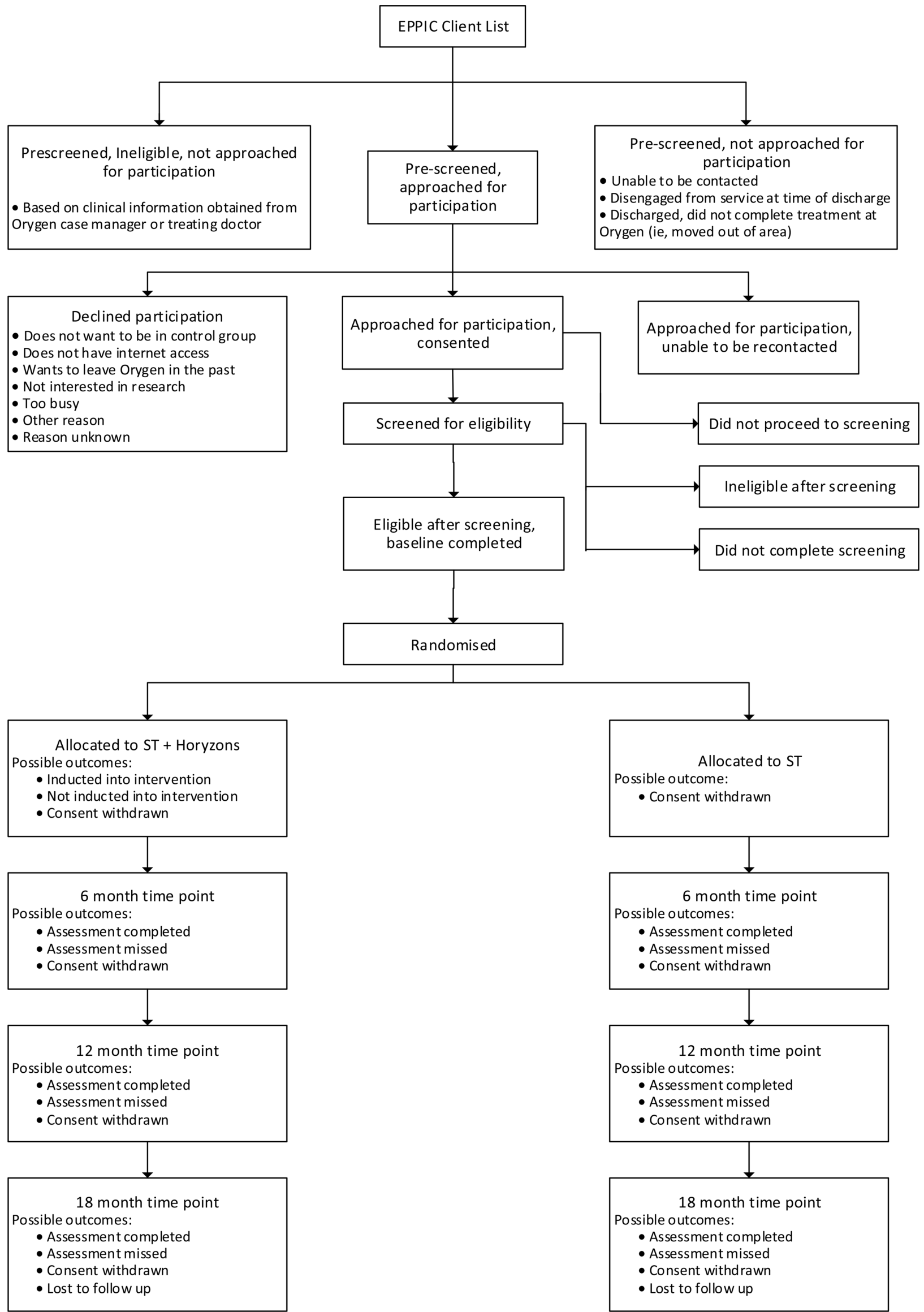

Figure 1 HORYZONS recruitment and allocation procedure. EPPIC, Early Psychosis Prevention and Intervention Centre; ST, standard treatment. 
Points'. To ensure that therapeutic content is translated into behavioural change, the Steps entail behavioural prompts entitled 'Do its'. For example, following a Step about fostering positive connections, the participant will find specific behavioural suggestions (or 'do its') to exercise a therapeutic skill (eg, empathy) in specific contexts (eg, school). 'Do its' are also related to the participant's specific strengths (eg, using kindness in social interactions). A 'Playlist' stores and schedules any 'Do it' the participant wants to complete in the future. Moreover, participants can rate, like, comment on, and share any Step or 'Do it' with others via the social networking newsfeed. Participants can also keep track of 'trending' Steps, or identify other young people who share their personal strengths. Finally, young people support each other's efforts to take on specific behavioural changes via the 'Team up' function (eg, by supporting or joining others in their efforts to take on specific challenges).

\section{Social network features}

Participants are encouraged to communicate with one another and with peer and expert moderators through the online social network or 'Café' to foster social support. Expert moderators (clinicians) are identifiable as a separate user class within the network. Each participant creates their own profile with images and can visit the wall of fellow users, where their posts and general activity are displayed. Posts can include 'icebreakers' (to encourage social interactions, eg, What's the worst gift that someone gave you?), user-generated threads, 'reactions' (designed to facilitate social support, eg, 'I get you' and 'thinking of you') as well as content related to mental health (eg, recent steps taken by others) or general interest.

A final feature of HORYZONS is Talk it out (TiO), an online group function informed by the evidence-based problem-solving framework. ${ }^{60} \mathrm{~A}$ TiO enables users to nominate issues (eg, 'how to break through shyness and make new friends?'), which are discussed in moderated groups through structured phases (eg, brainstorming, pros and cons and wrap-up). Previous problems and group solutions are stored in the system providing an easily accessible 'solution wiki' for future young people.

\section{Expert and peer moderation}

HORYZONS integrates online personal therapist support (by clinicians with experience treating young people with psychosis). Their role is to customise evidence-based interventions, monitor participant's clinical status and ensure the safety of the social network. Each therapist is assigned a caseload (ie, a $20 \%$ full-time equivalent online moderator can comfortably manage 20-25 participants), which they follow for the duration of the trial. Following the baseline assessment and initial face-to-face orientation to the system, the therapist makes contact with the participant for a brief phone meeting reviewing their personal needs and preferences. ${ }^{61}$ Expert moderators then develop brief case formulations that are presented during weekly supervision meetings with senior clinical psychologists from the team. Guided by the individual formulation, moderators send each client tailored content suggestions weekly (eg, a Step or 'Do it') with a focus on improving social functioning. Suggestions appear on the user's home page, and they receive a system notification, which is also delivered via SMS as determined by the participants settings. Young people can rate the helpfulness of the suggestions, which moderators use to tailor subsequent recommendations. Expert moderation was informed by the supportive accountability model ${ }^{61}$ a theory-driven framework operationalising how human support increases user engagement, the self-determination theory ${ }^{59}$ and strengths-based models ${ }^{62}$ as a means of enhancing users' engagement and self-efficacy.

In addition to clinical moderation, HORYZONS incorporates online vocational support. Drawing on our previous work, ${ }^{58}$ the vocational moderator provides individualised online vocational support, which can include: assessing young people's preferences and training, identifying suitable competitive job openings, supporting young people in specific job seeking activities (eg, writing a CV) or preparing for a job interview.

The 'cafe' is led by trained young people with lived experience of mental illness ('Peer-workers'). Peer workers are peer moderators who facilitate social learning using HORYZONS in desired ways (eg, self-disclosing, using therapy content to deal with difficulties). Peer-workers also seed discussion threads and 'icebreakers' to enable relevant, enjoyable conversations and facilitate meaningful relationships. Finally, peer moderation serves to normalise experiences, counteract stigma and promote engagement. Peer moderation was informed by the social learning theory that posits that those who observe others (ie, superusers) being rewarded for a particular behaviour (eg, completing a step or commenting on the social network) are more likely to modify their beliefs and subsequent behaviour. ${ }^{63}$

\section{Control intervention}

Participants randomised to regular care receive TAU following discharge from the EPPIC programme. TAU consists of a range of treatment options delivered by generic medical or mental health services typically available to young people in the absence of enrolment in the study. These can include follow-up by a general practitioner, private psychiatrist, primary care youth mental health services or adult mental health services that deliver multidisciplinary psychiatric care (including medical follow-up, case management and acute psychiatric care as appropriate). Prior to discharge from specialised FEP support, the EPPIC team, in collaboration with the young person, recommends the best treatment option based on the complexity of the young person's needs. Those with complex needs are referred to adult mental health services, while young people who attained a good level of recovery and remained stable are recommended primary care services. Additionally, TAU participants are provided with a printed leaflet containing relevant information 
on existing e-mental health resources for young people (ie, Moodgym, e-headspace, Reach-out and OYH Client's hub).

\section{Safety protocol}

The safety protocol is composed of three levels of security including: (1) system and privacy protection; (2) online safety; and (3) clinical safety. ${ }^{64}$

HORYZONS is hosted on a University of Melbourne web server. The University has industry standard measures in place to prevent unauthorised access to the server. The online system also integrates measures to secure the application and database against unauthorised access. These measures conform to industry best practice as defined by the Open Web Application Security Project. Privacy and online safety are managed in accordance with the Australian Communications and Media Authority.

The study coordinator carries out an initial face-to-face orientation with HORYZONS participants, including details of the terms of use. Participants were required to accept and comply with the guidelines for safe use of HORYZONS. When needed, participants are offered guidance on appropriate usage of the system. All users are asked to nominate an emergency contact person, such as a close family member. HORYZONS includes a 'report function' that enables young people to report a concern about any material posted by a user. The moderator assesses the basis of the report and responds accordingly, which may include the removal of the material and, in some cases, deactivating or restricting the young person's account. Participants are also able to hide their profile and activity should they become concerned about their privacy.

Clinical risk is managed through manual and automated procedures. First, moderators monitor the system twice daily on weekdays and once daily on weekends for evidence of clinical risk or deterioration. Any detected increased risk activates the HORYZONS crisis protocol that includes one or more of the following: a risk assessment with the young person, inform the research team, alert the emergency contact nominated by the participant and liaise with suitable emergency services where necessary. In addition, the system incorporates visible emergency guidelines and contact information. Finally, HORYZONS includes an automated keyword detection function, which activates each time a participant posts a contribution indicative of clinical risk or that contains potentially offensive words. The function blocks posts with notifications sent to the young person and the moderator, who can 'unblock' the post should they determine it to be unproblematic.

\section{Temporary withdrawal criteria}

In the event of a clinically significant deterioration of psychotic symptoms, increased risk or a hospital admission the clinical moderators perform an assessment to determine the risks and benefits of a temporary withdrawal from HORYZONS. Based on this assessment, and in consultation with the young person, the moderator team determines whether the account is temporarily suspended, or level of access is restricted. Following suspensions or restrictions to a user's account, the moderator will contact the young person at monthly intervals to ascertain whether the account is to be reactivated.

\section{Outcome measures}

Primary and secondary outcomes are measured at baseline (prior to randomisation), and at 6-month, 12-month and 18-month follow-up (table 1). Moreover, social functioning is tracked in real time for a period of 7 days after each assessment using ecological momentary assessment using a purpose-built smartphone application Smartphone Ecological Momentary Assessment (SEMA).

\section{Primary outcome}

The primary outcome measure is social functioning as measured by the Personal and Social Performance Scale (PSP) at 18-month follow-up. The PSP is a 100-point single-item rating scale derived from Social and Occupational Functioning Assessment Scale developed specifically to assess social functioning in schizophrenia. The PSP has shown strong psychometric properties ${ }^{6566}$ and has been recommended as one of the best existing tools to assess social functioning in psychosis. ${ }^{67}$

Additionally, with the purpose of capturing the full construct of social functioning, the First Episode Social Functioning Scale (FESFS) will be administered at each assessment time point.

The FESFS has been developed to measure social functioning in young people with FEP. ${ }^{68}$ Based on their psychometric properties and specific focus on social functioning, the following FESFS subscales were selected: friends and activities $(\alpha=0.80)$; independent living skills $(\alpha=0.81)$; interacting with people $(\alpha=0.80)$; and intimacy $(\alpha=0.75)$. These subscales have shown to correlate with other measures of social functioning, to be independent of psychotic symptoms and to be sensitive to treatment effects. ${ }^{68}$

\section{Secondary outcomes}

After the study was initiated, some feasibility issues were identified that led to modifications to the study secondary outcome measures. In the original protocol, we intended to measure psychotic relapse using the PANSS scale via phone or Skype-based assessments conducted every 2 months throughout the 18 -month intervention period. Ongoing measurement of psychotic symptoms at regular intervals is a requirement for the reliable and prospective identification of psychotic relapse. ${ }^{69}$ However, despite our best efforts, contacting participants via phone calls at regular intervals raised important feasibility issues, with many participants not answering phone calls or regularly changing phone numbers, leading to significant missing data. Thus, 12 months after study commencement, it was decided to discontinue the regular phone calls and prospective assessment of psychotic relapse. Given the 
Table 1 Schedule of outcome measures

Baseline

6 months

12 months 18 months

\section{Primary outcome}

Personal and Social Performance Scale

First Episode Social Functioning Scale

\section{Secondary outcomes}

Hospital admissions*

Calgary Depression Scale for Schizophrenia

Medical Outcomes Study: Social Support Survey

University of California, Los Angeles Loneliness Scale

Self-Esteem Rating Scale-Short Form

Depression Anxiety and Stress Scale

Mental Health Confidence Scale

Social Interaction Anxiety Scale

Scales of Psychological Well-being

Satisfaction with Life Scale

Assessment of Quality of Life - 8D questionnaire

The Positive and Negative Syndrome Scale

Employment and Education Status

Alcohol, Smoking, Substance Involvement Screening Test

Smartphone Ecological Momentary Assessment $†$

Resource Use Questionnaire

\section{Exploratory outcomes}

Social Comparison Scale

2-Way Social Support Scale

Savoring Beliefs Inventory

Mindful Attention Awareness Scale

Strengths Use Scale

Self-Compassion Scale Short Form

Physical Activity Questionnaire

Waist circumference

\section{Potential covariates}

Duration of Untreated Psychosis

Scale to Assess Unawareness of Mental Disorder

Motivational Trait Questionnaire

Medication Adherence Rating Scale

Bell Lysaker Emotion Recognition Task

The Hinting Task

Social Probabilistic Inference Task

Digit Symbol Substitution Test

Wechsler Test of Adult Reading

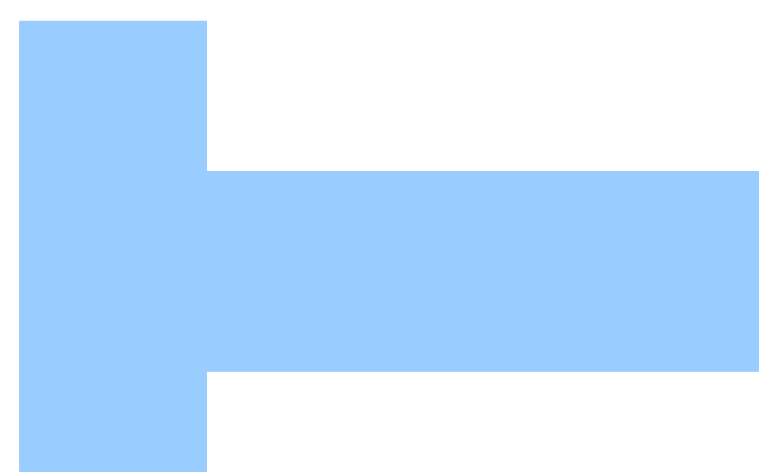

\section{HORYZONS-specific measures}

Horyzons Perceived Competence Scale

Horyzons Self-regulation Questionnaire

Horyzons Healthcare Climate Questionnaire

*Continuous from state government databases.

†Smartphone Ecological Momentary Assessment surveys. 
feasibility issues measuring relapse of psychotic symptoms at regular intervals, the following secondary outcomes were added:

1. Hospital admissions due to psychotic symptoms and mental health issues were added as a secondary outcome variable. We have access to reliable and objective hospital admission data from state databases (ie, Centre for Victorian Data Linkage) spanning the 18-month assessment period. Data on hospital admission from the state databases will be provided by an independent person blind to study design and purpose.

2. Positive and negative psychotic symptoms as measured by the PANNS scale at each assessment time point.

3. Physical health was also initially included as secondary outcome variable because we originally intended to incorporate online modules targeting this domain. However, we decided not to include therapy content addressing physical health and therefore this variable will be analysed as an exploratory outcome.

Secondary outcome measures include:

1. Accumulated hospital admissions due to psychotic symptoms and mental health issues over 18 months.

2. Vocational status as measured by employment and/or education status.

3. Depression as measured by the Calgary Depression Scale for Schizophrenia. ${ }^{70}$

4. Social support and loneliness as assessed by Medical Outcomes Study: Social Support Survey ${ }^{71}$ and the UCLA Loneliness Scale (Version $3^{72}$ ).

5. Self-esteem and self-efficacy as measured by the SelfEsteem Rating Scale-Short Form ${ }^{73}$ and Mental Health Confidence Scale ${ }^{74}$, respectively.

6. Anxiety and stress as determined by the Depression Anxiety and Stress Scale. ${ }^{75}$

7. Psychological well-being as measured by Scales of Psychological Well-being. ${ }^{76}$

8. Satisfaction with life as measured by Satisfaction with Life Scale. ${ }^{77}$

9. Quality of life as measured by the AQoL $8 \mathrm{D} .{ }^{78}$ This questionnaire can also be used to determine quality-adjusted life years (QALYs), which are useful in economic evaluation studies.

10. Positive and negative psychotic symptoms assessed by means of the PANSS. ${ }^{50}$

11. Substance use as measured by the Alcohol, Smoking and Substance Involvement Screening Test (version 3.1) over 18-month follow-up.

12. Cost-effective analysis: a Resource Use Questionnaire (RUQ) is used to determine the broader resource use of participants (eg, community mental health services, accommodation and work impacts). Additionally, for consenting participants, information regarding utilisation of healthcare services available via the Medicare Benefits Schedule (MBS; medical, allied health, diagnostic and pathology services) and the Pharmaceutical Benefits Schedule (PBS; medications) will be accessed from the Australian Department of Human Services.
To obtain more dynamic and ecologically valid data on young people's social functioning, participants use a custom-built smartphone app, SEMA, which is readily downloadable at no charge to participants owning a smartphone (running Android or iOS operating systems). SEMA delivers surveys (administered for 7 days following each assessment time point) approximately eight times per day for seven consecutive days. Young people are prompted to complete SEMA surveys at random times every $90 \mathrm{~min}( \pm 30 \mathrm{~min})$ over a 12-hour period (eg, 10:00to 22:00). SEMA tracks participants' responses in (near) real time, ensuring minimal data loss by uploading responses to a secure server or storing responses on the young person's smartphone when an internet connection is temporarily unavailable. Each SEMA survey begins with four items assessing momentary positive affect ('At the moment, how happy do you feel?', negative affect ('At the moment, how sad do you feel?'; 'At the moment, how stressed do you feel?') and momentary social isolation (eg, 'At the moment, how lonely do you feel?') rated on visual slider scales anchored at 0 (not at all) and 100 (very). The order of these four items is randomised at each survey. Following the momentary affect items, the SEMA survey includes items pertaining to social interactions of the young person (eg, 'How much time have you spent interacting with others, since last survey?'), perceived social efficacy (eg, 'How well do you think you handled your social interactions, since last survey?'), perceived social support (eg, 'have you received support or encouragement from others, since last survey?'), critical comments (eg, 'Have you felt that others criticized or judged you, since last survey') and social rank (eg, 'How competent have you felt in relation to others, since last survey?'). The order of these items is also randomised at each survey.

\section{Exploratory outcomes and potential covariates}

Additional exploratory outcomes included: social anxiety measured through the Social Interaction Anxiety Scale ${ }^{79}$ ; social comparison and group fit as assessed through the Social Comparison Scale ${ }^{80}$; the provision of emotional support measured via the 2-Way Social Support Scale ${ }^{81}$; anticipatory pleasure assessed through the Savoring Beliefs Inventory $^{82}$; mindfulness skills as assessed using the dispositional Mindful Attention Awareness Scale ${ }^{83}$; strengths use as assessed by means of the Strengths Use Scale ${ }^{84}$; self-compassion as assessed by the Self-Compassion Scale Short Form $^{85}$; physical health as measured by waist circumference over 18-month follow-up; and physical activity as measured by the International Physical Activity Questionnaire ${ }^{86}$ and by measuring sitting time across different domains ${ }^{87}$ (eg, $\mathrm{TV}$, video, computer and working).

Finally, potential covariates included: Duration of Untreated Psychosis defined as the time interval between onset of definite positive psychotic symptoms and first engagement and treatment in an early intervention (EI) service; clinical insight as assessed by means of the Scale to Assess Unawareness of Mental Disorder ${ }^{88}$; 
intrinsic motivation measured through the short form of Motivational Trait Questionnaire ${ }^{89}$; medication adherence measured by the Medication Adherence Rating Scale ${ }^{90}$; emotion processing assessed by means of the Bell Lysaker Emotion Recognition Task ${ }^{91}$; theory of mind measured using The Hinting Task ${ }^{92}$; jumping to conclusions measured through the Social Probabilistic Inference Task; premorbid intelligence as assessed via Wechsler Test of Adult Reading ${ }^{93}$; and general cognitive deficits will be measured through the Digit Symbol Substitution Test. ${ }^{94}$

\section{HORYZONS-specific measures}

Usage of HORYZONS is continuously monitored across the study intervention period (ie, frequency, duration and patterns of use). In addition, users complete self-report measures informed by the self-determination theory including: their perceived competence using the system, motivations for using it and their perception of moderation by HORYZONS.

\section{Statistical analysis and sample size}

Primary analyses will be undertaken on an intention-totreat basis. Mixed-model repeated measures (MMRM) analyses will be used to compare change in social functioning between the two treatment groups over the 18-month follow-up. MMRM is the analysis of choice because assumptions of traditional data analysis methods (eg, analysis of variance and regression) may be violated, such as the assumption of homogeneity of regression across time points. ${ }^{95}$ In addition, MMRM uses all available data (including participants with partial data) to estimate treatment effects. Time (baseline, 6, 12 and 18 months) will be the within-person predictor and treatment group (HORYZONS plus TAU vs TAU) the between-person predictor. MMRM will also be used to analyse change in the continuous secondary outcomes over 18 months. Additional analyses will use multiple imputation to assess the robustness of the findings to the choice of method for handling missing data. Ecological momentary assessment will be analysed using a multilevel structural equation modelling framework. ${ }^{96}$ Differential rate of hospital admissions will be analysed using multilevel logistic regression. Time to hospital admissions will be assessed by survival analysis (using either proportional hazard or accelerated lifetime models). Additional comparisons between treatment groups based on completers-only analyses will be conducted. Analyses will be undertaken in accordance with ICH 9 guidelines including a full analysis as well as per protocol set. The per protocol sample will be defined based on receiving a prespecified minimal exposure to the online intervention (ie, more than 8 logins over the 18-month intervention period).

Economic evaluation will comprise a cost-consequences analysis whereby incremental costs of the intervention will be compared with the full spectrum of study outcomes. A cost utility analysis will also be undertaken whereby the AQoL $8 \mathrm{D}$ will be used to QALYs. The evaluation will measure and value any change to the use of healthcare resources over the period of the study (using the data from the RUQ, MBS/PBS and hospitalisation administrative data) between the two treatment arms, and then compare any additional costs to the additional outcomes achieved. Australian sourced unit costs will be attached to the RUQ (from Australian sources such as the Commonwealth Department of Health, Mental Health Branch). Standardised economic evaluation techniques including incremental analysis of mean differences (using statistical techniques such as generalised linear models) and bootstrapping to determine confidence intervals around incremental cost-effectiveness ratios will be used. If, as expected, the intervention is found to be effective, lifetime and population cost-effectiveness of the interventions will be determined using economic modelling techniques. We will determine the likelihood that the intervention is cost-effective at commonly used value-formoney thresholds such as $\$ 20000 /$ QALY and $\$ 50000$ / QALY.

The primary outcome is change in social functioning at 18-month follow-up. A recent RCT investigating the effects of extending FEP specialist treatment for 12 months (ie, a total of 3 years of specialist treatment) reported an effect size of 0.53 (Cohen's $d$ ) for functional outcomes for the extended model of care at 12 months (ie, end of the specialised treatment) compared with TAU (ie, 2 years of specialist treatment) ${ }^{32}$ If we assume that alpha is set at 0.05 and power $(1-\beta)$ at 0.90 , then a sample size of 70 is required for each of the two groups (total $\mathrm{n}=140)$ to detect medium effect sizes (0.5; Cohen's $d$ ). For the second outcome measure of hospital admissions at 18 months follow-up, there will be $80 \%$ power to detect an improvement in the rate of hospital admissions of at least $43 \%$ in the TAU+HORYZONS, assuming a hospital admission rate in the TAU of $30 \%$ over the 18-month follow-up. ${ }^{2}$ We recruited 170 participants, accommodating for an $18 \%$ attrition rate, which is consistent with a similar study in terms of design and population. ${ }^{32}$

\section{Data management}

A custom-built online RPMS is used to manage the electronic data from this study. The RPMS includes an electronic case report form (eCRF) and randomisation functionality. The study assessors record participant-level data on a paper-based case report form. These data are subsequently entered into the eCRF section of the RPMS. The randomisation functionality of the RPMS is operated by the study coordinator. The RPMS is accessed using a secure website and is stored on a secure server. It is designed to maintain the privacy and confidentiality of participant information and to ensure the integrity of the data. Access to RPMS is restricted to study personnel, and the level of access is dependent on the person's role. The study assessors and investigators do not have access to the randomisation section to ensure that they remain blind. Data are stored on three separate secure computer servers, including data collected from the SEMA tool, the RPMS and data accumulated from participant activity 
within the HORYZONS online system. These various data are aggregated into a single electronic secure databank.

Data verification at all assessment time points is being conducted on 20 randomly selected cases. The selected cases are re-entered by the study coordinator. The a priori acceptable error rate has been set at $0.5 \%$.

\section{Ethics and dissemination}

All trial participants provided written informed consent prior to enrolment in the trial. For all eligible participants under 18 years of age, parental or guardian consent was also obtained.

Any adverse events (eg, hospital admissions) including an independent assessment of whether the adverse event was related to the online intervention (ie, made by a psychiatrist) were reported to the Melbourne Health Research and Ethics Committee. The study was considered to be low risk by the study sponsor and a trial management group was established in place of a data monitoring committee.

The main results of this clinical trial will be published in a peer-reviewed scientific journal. Manuscripts will also be prepared for significant findings regarding the secondary and exploratory aims. These results will be submitted and presented at scientific forums including national and international conferences in schizophrenia, early psychosis and youth mental health.

\section{Patient and public statement}

Patients were included in the development of the research questions and outcome measures in a number of ways. First, Orygen includes a youth reference group that provides consultation on the design, conduct and ethics of all studies carried out within the organisation. This group provided input into the main research question, design and outcome measures of the RCT. In addition, Orygen's internal Research and Review Committee integrates two youth representatives that also provided feedback on the key methodological aspects of the study from the consumers' perspective.

Second, the design, development and therapeutic content of the intervention was also designed in partnership with young people. We conducted a series of focus groups with young people with lived experience to inform the development of HORYZONS. Young people participating in these focus groups consistently stated that HORYZONS should focus on promoting social connectedness and personal strengths. ${ }^{35}{ }^{36}$ This is consistent with previous qualitative research with young people. ${ }^{97}$ The outcome measures were selected based on the combination of this research and this feedback. However, we did not seek specific assessment from young people on the burden of assessments or the intervention. Given that participants can select the frequency with which they use the system and receive contact from the moderator team, this seemed less salient with respect to the intervention.

Patients were not involved in the recruitment into the study. However, peer workers were involved in the conduct of the online intervention. We established a peer workers reference group led by our youth participation coordinator. This group provided online peer support via HORYZONS as well as ongoing consultation on the management of the trial and intervention updates. In addition, a number of focus groups with participants from the HORYZONS trial were conducted to obtain feedback on the management (moderation) and content of the online system.

We have created an email list to inform all participants of the results of the study and provided a contact email for participants to contact the research team should they require any additional information or wish to participate in online peer support.

\section{DISCUSSION}

The onset of psychosis often strikes young people at the prime of their lives, triggering a myriad of adverse psychosocial consequences that can result in entrenched social isolation, unemployment and chronicity. ${ }^{3}$ Against this, early intervention is now seen as a key strategy to improve long-term recovery and reduce treatment costs. ${ }^{3}$ However, while specialist early psychosis services have been demonstrated that they improve outcomes in FEP, follow-up studies have questioned the maintenance of treatment effects beyond the intervention period. ${ }^{9} 10$ Moreover, social recovery, a priority for young people, continues to be resistant to current intervention approaches. ${ }^{19}$ This is the first RCT to evaluate a novel online social media intervention designed to address both these challenges.

HORYZONS is the first intervention to exploit online social media technology and apply strengths and mindfulness approaches to improve long-term social recovery in FEP. In addition, the design of the intervention builds on our extensive experience developing and evaluating effective relapse prevention ${ }^{98-100}$ and vocational recovery interventions $^{58}$ in early psychosis. Thus, HORYZONS weaves together two novel intervention approaches for FEP with established evidence-based protocols, while drawing on a strong theoretical base for social recovery in early psychosis (ie, self-determination theory, ${ }^{59}$ broaden and build theory ${ }^{22}$.

Building on a previous successful pilot study, ${ }^{36}$ HORYZONS was codeveloped with end-users and service providers. The online system was designed to be scalable, embedded within clinical practice and delivered across early intervention services. Specifically, HORYZONS is moderated by EPPIC clinicians as part of their routine clinical role (ie, clinicians would allocate a proportion of their clinical time, typically $20 \%$ to $30 \%$, to online moderation). Moderation and training procedures have been manualised and require minimum specialised training (2 days). Therapist efficiency using HORYZONS is estimated to be five times higher than that of specialised FEP services (100 vs 20 young people of a typical caseload in an early psychosis clinic). Thus, if successful, HORYZONS will provide a scalable, cost-effective intervention 
approach to extend the benefits of early intervention and improve social functioning in patients with FEP.

A limitation of the current study is that the control intervention consists of routine care, as opposed to a sham intervention accounting for increased attention and unspecific therapeutic factors. That said, this decision was made to enhance the external validity of the findings by replicating the current mainstream follow-up options available to FEP young people beyond their involvement in early intervention services. As such, this study is expected to provide evidence of cost-effectiveness of a step-down model of care instead of generating controlled evidence on the specific treatment components driving improved outcomes. Of note, the design of this study parallels that of recently published RCTs examining extended interventions for FEP services, with TAU being the control intervention across all three studies. ${ }^{30-32}$

Sustained and meaningful recovery is the ultimate goal of early intervention services as well as the most valued outcome by young people and their families. ${ }^{101}$ This is the first RCT trial to evaluate an online-based intervention as a means to extend the benefits of specialised early intervention services and foster long-term social functioning in FEP. Thus, if successful, HORYZONS has the potential to augment the benefits and long-term impact of the current model of early intervention for psychosis.

\section{Author affiliations}

${ }^{1}$ Orygen, The National Centre of Excellence in Youth Mental Health, Melbourne, Victoria, Australia

${ }^{2}$ Centre for Youth Mental Health, The University of Melbourne, Parkville, Victoria, Australia

${ }^{3}$ Melbourne School of Psychological Sciences, University of Melbourne, Melbourne, Victoria, Australia

${ }^{4}$ School of Computing and Information Systems, University of Melbourne, Parkville, Victoria, Australia

${ }^{5}$ School of Behavioural and Health Sciences, Australian Catholic University,

Melbourne, Victoria, Australia

${ }^{6}$ Department of Psychology, University of North Carolina Chapel Hill, NC, United Stated

${ }^{7}$ Hotchkiss Brain Institute, Department of Psychiatry, University of Calgary, Calgary, Alberta, Calgary, Alberta, Canada

${ }^{8}$ School of Health and Social Development, Deakin University, Geelong, Victoria, Australia

${ }^{9}$ University Hospital Marques de Valdecilla-IDIVAL, Santander, Spain

${ }^{10}$ School of Rehabilitation, Faculty of Medicine, University of Montreal, Montreal, Quebec, Canada

${ }^{11}$ University of Montreal Hospital Research Center, Health Innovation and Evaluation Hub, Montreal, Quebec, Canada

${ }^{12}$ Douglas Mental Health University Institute, Montreal, Quebec, Canada

${ }^{13}$ Monash University, Melbourne, Victoria, Australia

${ }^{14}$ University Hospital 'Virgen del Rocio', Seville, Spain

Acknowledgements The authors wish to thank the Orygen Youth Advisory Council and Orygen Youth Research Council for their input into the development of Horyzons. The authors would like to thank the peer workers (Damian, Mabigail, Samantha, Kay, Stuart, Amal, Margie, Megan, Gene, Matt, Booma, Nicole, Rebecca, Elise, Emily, Sarah, Ally, Matt, Joanne, Imesha and Tricia) and online moderators (Catherine Spillane, Jasmin Watson, Lisa Rumney, Sandra Seif, Jacqui Mackinnon, Edwina Ford, Gina Chinnery, Dylan Alexander, Rebecca Davenport, Nerida Barclay, Jennifer Butler, Melanie Cooke and Matthew Stuckey) for their valuable contributions to the study. The authors also wish to thank the inspiring and generous young people who took part in the study.
Contributors MA-J and JFG led the overall design and conduct of the study. SB and SR contributed to the supervision of the moderation of the online intervention. PK is the lead statistics and ecologically momentary expert in the study. JP was the peer workers supervisor during the study. SD is the lead engineer of the HORYZONS project. ChM is the lead front-end designer of the HORYZONS platform. PR is the creative content lead of the project. RL, GW, OS-E, TG and CL contributed to the design of the intervention. $\mathrm{RC}$ developed the mindfulness and self-compassion components of HORYZONS. MA-J wrote the first draft of the manuscript. DC, LV, CM, DLP, HH, CG-B, RD-G, EK, SMC, SL and PDM contributed to the design and conduct of the study. All authors critically revised and approved the final manuscript.

Funding The HORYZONS trial was supported by the Mental IIIness Research Fund from the State Government of Victoria. MA-J was supported by a Career Development Fellowship (APP1082934) from the National Health and Medical Research Council (NHMRC). SMC has been supported by a Career Development Fellowship (APP1061998) and Senior Research Fellowship (APP1136344) from NHMRC. ChM was supported by a NHMRC Early Career Fellowship (APP1035887) during the conduct of the trial. SL was supported in part by a New Investigator Salary Award from the Canadian Institutes of Health Research and previously in part by a Research Scholar Salary Award from the Fonds de recherche du Québec-Santé.

Competing interests None declared.

Patient consent Not required.

Ethics approval Ethics approval for the trial was provided by the Melbourne Health Research and Ethics Committee (№. 2013.146).

Provenance and peer review Not commisioned; peer reviewed for ethical and funding approval prior to submission.

Open access This is an open access article distributed in accordance with the Creative Commons Attribution Non Commercial (CC BY-NC 4.0) license, which permits others to distribute, remix, adapt, build upon this work non-commercially, and license their derivative works on different terms, provided the original work is properly cited, appropriate credit is given, any changes made indicated, and the use is non-commercial. See: http://creativecommons.org/licenses/by-nc/4.0/.

\section{REFERENCES}

1. Psychiatrists RAaNZCo. The economic cost of serious mental illnessand comorbidities in Australia and New Zealand, 2016.

2. Alvarez-Jimenez M, Priede A, Hetrick SE, et al. Risk factors for relapse following treatment for first episode psychosis: a systematic review and meta-analysis of longitudinal studies. Schizophr Res 2012;139(1-3):116-28.

3. McGorry PD, Killackey E, Yung A. Early intervention in psychosis: concepts, evidence and future directions. World Psychiatry 2008;7:148-56.

4. Alvarez-Jiménez M, Parker AG, Hetrick SE, et al. Preventing the second episode: a systematic review and meta-analysis of psychosocial and pharmacological trials in first-episode psychosis. Schizophr Bull 2011;37:619-30.

5. Bird V, Premkumar P, Kendall T, et al. Early intervention services, cognitive-behavioural therapy and family intervention in early psychosis: systematic review. Br J Psychiatry 2010;197:350-6.

6. Petersen $L$, Jeppesen $\mathrm{P}$, Thorup $\mathrm{A}$, et al. A randomised multicentre trial of integrated versus standard treatment for patients with a first episode of psychotic illness. BMJ 2005;331:602.

7. Craig TK, Garety P, Power P, et al. The Lambeth Early Onset (LEO) Team: randomised controlled trial of the effectiveness of specialised care for early psychosis. BMJ 2004;329:1067.

8. Melle I, Larsen TK, Haahr U, et al. Reducing the duration of untreated first-episode psychosis: effects on clinical presentation. Arch Gen Psychiatry 2004;61:143-50.

9. Bertelsen M, Jeppesen P, Petersen L, et al. Five-year follow-up of a randomized multicenter trial of intensive early intervention vs standard treatment for patients with a first episode of psychotic illness: the OPUS trial. Arch Gen Psychiatry 2008;65:762-71.

10. Gafoor R, Nitsch D, McCrone P, et al. Effect of early intervention on 5-year outcome in non-affective psychosis. Br J Psychiatry 2010;196:372-6.

11. Alvarez-Jimenez M, O'Donoghue B, Thompson A, et al. Beyond Clinical Remission in First Episode Psychosis: Thoughts on Antipsychotic Maintenance vs. Guided Discontinuation in the Functional Recovery Era. CNS Drugs 2016;30:357-68.

12. Horan WP, Subotnik KL, Snyder KS, et al. Do recent-onset schizophrenia patients experience a "social network crisis"? Psychiatry 2006;69:115-29. 
13. Macdonald EM, Hayes RL, Baglioni AJ. The quantity and quality of the social networks of young people with early psychosis compared with closely matched controls. Schizophr Res 2000;46:25-30.

14. Morgan C, Kirkbride J, Hutchinson G, et al. Cumulative social disadvantage, ethnicity and first-episode psychosis: a case-control study. Psychol Med 2008;38:1701-15.

15. Sündermann $O$, Onwumere $J$, Kane $F$, et al. Social networks and support in first-episode psychosis: exploring the role of loneliness and anxiety. Soc Psychiatry Psychiatr Epidemiol 2014;49:359-66.

16. Norman RM, Malla AK, Manchanda R, et al. Social support and three-year symptom and admission outcomes for first episode psychosis. Schizophr Res 2005;80(2-3):227-34.

17. Norman RM, Windell D, Manchanda R, et al. Social support and functional outcomes in an early intervention program. Schizophr Res 2012;140(1-3):37-40.

18. Howard L, Leese M, Thornicroft G. Social networks and functional status in patients with psychosis. Acta Psychiatr Scand 2000;102:376-85.

19. Fowler D, Hodgekins J, French P, et al. Social recovery therapy in combination with early intervention services for enhancement of social recovery in patients with first-episode psychosis (SUPEREDEN3): a single-blind, randomised controlled trial. Lancet Psychiatry 2018:5:41-50.

20. Browne J, Estroff SE, Ludwig K, et al. Character strengths of individuals with first episode psychosis in Individual Resiliency Training. Schizophr Res 2018:195:448-54.

21. Vaskinn A, Ventura J, Andreassen OA, et al. A social path to functioning in schizophrenia: From social self-efficacy through negative symptoms to social functional capacity. Psychiatry Res 2015;228:803-7.

22. Garland EL, Fredrickson B, Kring AM, et al. Upward spirals of positive emotions counter downward spirals of negativity: insights from the broaden-and-build theory and affective neuroscience on the treatment of emotion dysfunctions and deficits in psychopathology. Clin Psychol Rev 2010;30:849-64.

23. Garland EL, Geschwind N, Peeters F, et al. Mindfulness training promotes upward spirals of positive affect and cognition: multileve and autoregressive latent trajectory modeling analyses. Front Psychol 2015;6:15.

24. Roe D, Hasson-Ohayon I, Mashiach-Eizenberg M, et al. Narrative enhancement and cognitive therapy (NECT) effectiveness: a quasiexperimental study. J Clin Psychol 2014;70:303-12.

25. Hall PL, Tarrier N. The cognitive-behavioural treatment of low self-esteem in psychotic patients: a pilot study. Behav Res Ther 2003:41:317-32.

26. Linszen D, Dingemans $P$, Lenior $M$. Early intervention and a five year follow up in young adults with a short duration of untreated psychosis: ethical implications. Schizophr Res 2001;51:55-61.

27. Norman RM, Manchanda R, Malla AK, et al. Symptom and functional outcomes for a 5 year early intervention program for psychoses. Schizophr Res 2011;129(2-3):111-5.

28. Crumlish N, Whitty P, Clarke M, et al. Beyond the critical period: longitudinal study of 8-year outcome in first-episode non-affective psychosis. Br J Psychiatry 2009;194:18-24.

29. Álvarez-Jiménez M, Gleeson JF, Henry LP, et al. Road to full recovery: longitudinal relationship between symptomatic remission and psychosocial recovery in first-episode psychosis over 7.5 years. Psychol Med 2012;42:595-606.

30. Albert N, Melau M, Jensen $\mathrm{H}$, et al. Five years of specialised early intervention versus two years of specialised early intervention followed by three years of standard treatment for patients with a first episode psychosis: randomised, superiority, parallel group trial in Denmark (OPUS II). BMJ 2017;356:i6681.

31. Malla $A$, Joober R, lyer $\mathrm{S}$, et al. Comparing three-year extension of early intervention service to regular care following two years of early intervention service in first-episode psychosis: a randomized single blind clinical trial. World Psychiatry 2017;16:278-86.

32. Chang WC, Kwong VWY, Lau ESK, et al. Sustainability of treatment effect of a 3-year early intervention programme for first-episode psychosis. Br J Psychiatry 2017;211:37-44.

33. Singh SP. Early intervention in psychosis. Br J Psychiatry 2010;196:343-5.

34. Alvarez-Jimenez M, Alcazar-Corcoles MA, González-Blanch C, et al. Online, social media and mobile technologies for psychosis treatment: a systematic review on novel user-led interventions. Schizophr Res 2014;156:96-106.

35. Álvarez-Jiménez M, Gleeson JF, Bendall S, et al. Internet-based interventions for psychosis: a sneak-peek into the future. Psychiatr Clin North Am 2012;35:735-47.
36. Alvarez-Jimenez M, Bendall S, Lederman R, et al. On the HORYZON: moderated online social therapy for longterm recovery in first episode psychosis. Schizophr Res 2013:143:143-9.

37. Sensis. Sensis Social Media Report 2017 Chapter 1 - Australians and social media SENSIS. 22, 2017.

38. Duggan M, Ellison N, Lampe C, et al. Social Media Update: Pew Research Center Report, 2015:1-18.

39. Best P, Manktelow R, Taylor B. Online communication, social media and adolescent wellbeing: A systematic narrative review. Child Youth Serv Rev 2014;41:27-36.

40. Valenzuela S, Park N, Kee KF. Is There Social Capital in a Social Network Site?: Facebook Use and College Students' Life Satisfaction, Trust, and Participation. Journal of Computer-Mediated Communication 2009;14:875-901.

41. Deters FG, Mehl MR. Does Posting Facebook Status Updates Increase or Decrease Loneliness? An Online Social Networking Experiment. Soc Psychol Personal Sci 2013;4:579-86.

42. Verduyn P, Lee DS, Park J, et al. Passive Facebook usage undermines affective well-being: Experimental and longitudinal evidence. J Exp Psychol Gen 2015;144:480-8.

43. Birnbaum ML, Rizvi AF, Confino J, et al. Role of social media and the Internet in pathways to care for adolescents and young adults with psychotic disorders and non-psychotic mood disorders. Early Interv Psychiatry 2017:11:290-5.

44. Lal S, Dell'Elce J, Malla AK. Technology Access and Use Among Young Adults With a First Episode of Psychosis. Psychiatr Serv 2015;66:764-5.

45. Lal S, Dell'Elce J, Tucci N, et al. Preferences of Young Adults With First-Episode Psychosis for Receiving Specialized Mental Health Services Using Technology: A Survey Study. JMIR Ment Health 2015;2:e18.

46. Theraputic Goods Administration. Note for Guidance on Good Clinical Practice (CPMP/ICH/135/95), Annotated with TGA Comments. Canberra: Commonwealth Department of Health and Aged Care, 2000

47. Eysenbach G. CONSORT-EHEALTH Group. CONSORT-EHEALTH: improving and standardizing evaluation reports of Web-based and mobile health interventions. J Med Internet Res 2011;13:e126.

48. Chan AW, Tetzlaff JM, Altman DG, et al. SPIRIT 2013 statement: defining standard protocol items for clinical trials. Ann Intern Med 2013;158:200-7.

49. McGorry PD, Edwards J, Mihalopoulos C, et al. EPPIC: an evolving system of early detection and optimal management. Schizophr Bull 1996;22:305-26.

50. Kay SR, Fiszbein A, Opler LA. The positive and negative syndrome scale (PANSS) for schizophrenia. Schizophr Bull 1987;13:261-76.

51. Overall JE, Gorham DR. The Brief Psychiatric Rating Scale. Psycho Rep 1962;10:799-812.

52. First MB, Spitzer RL, Gibbon M, et al. Structured Clinical Interview for DSM-IV-TR Axis I Disorders. Research Version, Patient Edition: SCID-I/P. New York: Biometrics Research, New York State Psychiatric Institute, 2002.

53. First MB, Gibbon M, Spitzer RL, et al. User's guide for the structured clinical interview for DSM-IV axis II personality disorders: SCID-II: American Psychiatric Puba, 1997.

54. Lewis JA. Statistical principles for clinical trials (ICH E9): an introductory note on an international guideline. Stat Med 1999;18:1903-42.

55. Lederman R, Wadley G, Gleeson J, et al. Moderated online social therapy: designing and evaluating technology for mental health. ACM Transactions on Computer-Human Interaction 2014;21.

56. Alvarez-Jimenez M, Gleeson JF, Rice S, et al. Online peer-to-peer support in youth mental health: seizing the opportunity. Epidemio Psychiatr Sci 2016;25:123-6.

57. Beck AT, Grant PM, Huh GA, et al. Dysfunctional attitudes and expectancies in deficit syndrome schizophrenia. Schizophr Bull 2013;39:43-51.

58. Killackey E, Allott K, Cotton SM, et al. A randomized controlled trial of vocational intervention for young people with first-episode psychosis: method. Early Interv Psychiatry 2013;7:329-37.

59. Ryan RM, Deci EL. Self-determination theory and the facilitation of intrinsic motivation, social development, and well-being. Am Psychol 2000;55:68-78.

60. D'Zurilla TJ, Goldfried MR. Problem solving and behavior modification. J Abnorm Psychol 1971;78:107-26.

61. Mohr DC, Cuijpers P, Lehman K. Supportive accountability: a model for providing human support to enhance adherence to eHealth interventions. J Med Internet Res 2011;13:e30.

62. Seligman MEP, Rashid T, Parks AC. Positive psychotherapy. Am Psychol 2006;61:774-88. 
63. Bandura A. Social cognitive theory: an agentic perspective. Annu Rev Psychol 2001;52:1-26.

64. Gleeson JF, Lederman R, Wadley G, et al. Safety and privacy outcomes from a moderated online social therapy for young people with first-episode psychosis. Psychiatr Serv 2014;65:546-50.

65. Nasrallah H, Morosini P, Gagnon DD. Reliability, validity and ability to detect change of the Personal and Social Performance scale in patients with stable schizophrenia. Psychiatry Res 2008;161:213-24.

66. Gigantesco A, Vittorielli M, Pioli R, et al. The VADO approach in psychiatric rehabilitation: a randomized controlled trial. Psychiatr Serv 2006;57:1778-83.

67. Burns T, Patrick D. Social functioning as an outcome measure in schizophrenia studies. Acta Psychiatr Scand 2007;116:403-18.

68. Lecomte $\mathrm{T}$, Corbière $\mathrm{M}$, Ehmann $\mathrm{T}$, et al. Development and preliminary validation of the First Episode Social Functioning Scale for early psychosis. Psychiatry Res 2014;216:412-7.

69. Gleeson JF, Alvarez-Jimenez M, Cotton SM, et al. A systematic review of relapse measurement in randomized controlled trials of relapse prevention in first-episode psychosis. Schizophr Res 2010;119(1-3):79-88

70. Addington D, Addington J, Maticka-Tyndale E. Assessing depression in schizophrenia: the Calgary Depression Scale. $\mathrm{Br} \mathrm{J}$ Psychiatry Suppl 1993;163:39-44.

71. Sherbourne CD, Stewart AL. The MOS social support survey. Soc Sci Med 1991:32:705-14.

72. Russell DW. UCLA Loneliness Scale (Version 3): reliability, validity, and factor structure. J Pers Assess 1996;66:20-40.

73. Lecomte $T$, Corbière $M$, Laisné $F$. Investigating self-esteem in individuals with schizophrenia: relevance of the Self-Esteem Rating Scale-Short Form. Psychiatry Res 2006;143:99-108.

74. Castelein S, van der Gaag M, Bruggeman R, et al. Measuring empowerment among people with psychotic disorders: a comparison of three instruments. Psychiatr Serv 2008;59:1338-42.

75. Lovibond SH, Lovibond PF. Manual for the Depression Anxiety Stress Scales. 2nd. edn. Sydney: Psychology Foundation, 1995.

76. Ryff CD. Happiness is everything, or is it? Explorations on the meaning of psychological well-being. J Pers Soc Psychol 1989;57:1069-81.

77. Diener E, Emmons RA, Larsen RJ, et al. The satisfaction with life scale. J Pers Assess 1985;49:71-5.

78. Richardson J, Elsworth G, Lezzi A, et al. Increasing the sensitivity of the AQoL inventory for the evaluation of interventions affecting mental health. Melbourne: Centre for Health Economics, Monash University, 2011.

79. Mattick RP, Clarke JC. Development and validation of measures of social phobia scrutiny fear and social interaction anxiety. Behav Res Ther 1998;36:455-70.

80. Allan S, Gilbert P. A social comparison scale: Psychometric properties and relationship to psychopathology. Pers Individ Dif 1995;19:293-9.

81. Shakespeare-Finch J, Obst PL. The development of the 2-Way Social Support Scale: a measure of giving and receiving emotional and instrumental support. J Pers Assess 2011;93:483-90.

82. Bryant FB, Inventory SB. SBI: A scale for measuring beliefs about savouring. Journal of Mental Health 2003;12:175-96.
83. Brown KW, Ryan RM. The benefits of being present: mindfulness and its role in psychological well-being. J Pers Soc Psychol 2003;84:822-48.

84. Wood AM, Linley PA, Maltby J, et al. Using personal and psychological strengths leads to increases in well-being over time: A longitudinal study and the development of the strengths use questionnaire. Pers Individ Dif 2011;50:15-19.

85. Raes F, Pommier E, Neff KD, et al. Construction and factorial validation of a short form of the Self-Compassion Scale. Clin Psychol Psychother 2011;18:250-5.

86. Craig CL, Marshall AL, Sjöström M, et al. International physical activity questionnaire: 12 -country reliability and validity. Med Sci Sports Exerc 2003;35:1381-95.

87. Marshall AL, Miller YD, Burton NW, et al. Measuring total and domain-specific sitting: a study of reliability and validity. Med Sci Sports Exerc 2010;42:1094-102.

88. Amador XF, Strauss DH, Yale SA, et al. Assessment of insight in psychosis. Am J Psychiatry 1993;150:873-9.

89. Kanfer R, Ackerman P. Individual differences in work motivation: Further explorations of a trait framework. Appl Psychol 2000;49:470-82.

90. Thompson K, Kulkarni J, Sergejew AA. Reliability and validity of a new Medication Adherence Rating Scale (MARS) for the psychoses. Schizophr Res 2000;42:241-7.

91. Bell M, Bryson G, Lysaker P. Positive and negative affect recognition in schizophrenia: a comparison with substance abuse and normal control subjects. Psychiatry Res 1997;73(1-2):73-82.

92. Corcoran R, Mercer G, Frith CD. Schizophrenia, symptomatology and social inference: investigating "theory of mind" in people with schizophrenia. Schizophr Res 1995;17:5-13.

93. Ginsberg J. Wechsler test of adult reading. Appl Neuropsychol 2003;10:182-4.

94. Lezak MD. Neuropsychological assessment. USA: Oxford University Press, 2004.

95. Brown H, Prescott R. Applied mixed models in medicine. Statistics in Practice. 3 edn. Chichester: John Wiley \& Sons Inc, 2015.

96. Gleeson J, Lederman R, Herrman $\mathrm{H}$, et al. Moderated online social therapy for carers of young people recovering from first-episode psychosis: study protocol for a randomised controlled trial. Trials 2017; $18: 27$

97. Iyer SN, Mangala R, Anitha J, et al. An examination of patientidentified goals for treatment in a first-episode programme in Chennai, India. Early Interv Psychiatry 2011;5:360-5.

98. Gleeson JF, Cotton SM, Alvarez-Jimenez M, et al. A randomized controlled trial of relapse prevention therapy for first-episode psychosis patients. J Clin Psychiatry 2009;70:477-86.

99. Gleeson JF, Cotton SM, Alvarez-Jimenez M, et al. A randomized controlled trial of relapse prevention therapy for first-episode psychosis patients: outcome at 30-month follow-up. Schizophr Bull 2013;39:436-48.

100. Alvarez-Jiménez M, Gleeson JF, Cotton S, et al. Predictors of adherence to cognitive-behavioural therapy in first-episode psychosis. Can J Psychiatry 2009;54:710-8.

101. Santesteban-Echarri O, Paino M, Rice S, et al. Predictors of functional recovery in first-episode psychosis: A systematic review and meta-analysis of longitudinal studies. Clin Psychol Rev 2017;58:59-75. 